\title{
Non-thermal hybrid drying of fruits and vegetables: a review of current technologies
}

\begin{abstract}
Fruits and vegetables are very perishable commodities that have enormous industrial and commercial importance. To preserve its quality attributes, increase shelf life, and reduce transport weight, fruit and vegetable can be processed by drying. Over the years, conventional drying techniques have been widely applied, both industrially and commercially for the processing and preservation of fruits and vegetables. However, most of the conventional techniques are time and energy consuming, and affect important quality attributes of final products. Recently, there has been increased interest in the use of non-thermal drying methods in combination with other conventional drying techniques for preserving fruits and vegetables. These methods have been reported to enhance the quality attributes of dried products, reduce drying time and energy demand, and increase the overall drying efficiency. In this regard, the development of a cost effective non-thermal hybrid drying systems, such as combined ultrasound (US) and hot-air drying, ultraviolet (UV) and hot-air drying, and pulse electric field (PEF) and hot-air drying have recently been researched on. These drying techniques have become potential substitute for conventional industrial and commercial dryers, owing to their advantage of producing quality dried fruit and vegetable products, with reduced drying time and energy consumption. This study therefore attempts to highlight recent developments of the commonly used non-thermal combined convective hot-air drying (CHAD) techniques for fruits and vegetables, with emphasis on drying time, drying rate, quality attributes of products, and modelling approach. This study further highlights the primary constraints for industrial application of this technology as the inadequate medium of transmission for power ultrasound, cost of design and installation, and the limited study on (UV) and PEF assisted CHAD. The necessity for conducting more detailed studies on non-thermal assisted convective hot-air drying of fruits and vegetables was emphasized.
\end{abstract}

Keyword: Non-thermal technology; Hot-air assisted drying; Fruits and vegetables; Product quality; Modelling techniques 